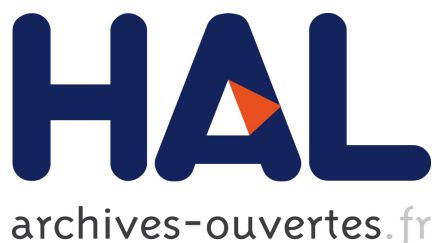

archives-ouvertes

\title{
Are users better-off with new transit lines?
}

Moez Kilani, André de Palma, Stef Proost

\section{To cite this version:}

Moez Kilani, André de Palma, Stef Proost. Are users better-off with new transit lines?. 2016. hal01282877

\section{HAL Id: hal-01282877 \\ https://hal-ens.archives-ouvertes.fr/hal-01282877}

Submitted on 14 Mar 2016

HAL is a multi-disciplinary open access archive for the deposit and dissemination of scientific research documents, whether they are published or not. The documents may come from teaching and research institutions in France or abroad, or from public or private research centers.
L'archive ouverte pluridisciplinaire HAL, est destinée au dépôt et à la diffusion de documents scientifiques de niveau recherche, publiés ou non, émanant des établissements d'enseignement et de recherche français ou étrangers, des laboratoires publics ou privés. 


\title{
Are users better-off with new transit lines?
}

\author{
Moez Kilani ${ }^{1}$, André de Palma ${ }^{2,3}$ and Stef Proost ${ }^{3}$ \\ ${ }^{1}$ LEM-CNRS (UMR 9221), University of Lille, France \\ ${ }^{2}$ École Normale Supérieure de Cachan, 61 Avenue du Prsident Wilson, \\ 94230 Cachan, France \\ ${ }^{3} \mathrm{KU}$ Leuven, Belgium
}

March 1, 2016

\begin{abstract}
This paper studies the entry of new competitors in public transport like long distance busses that compete with rail. A stylized model is used that analyzes the addition of a new more direct line in an existing network. We show that the introduction of the new line is only beneficial if there are relatively many users that want to use the new direct line. Our result raises serious concerns with respect to the decentralized management of transit systems.
\end{abstract}

JEL codes: R42; R48; H42

KEYWORDS: Transport investment, public transport, externalities 


\section{Introduction}

Over the last 20 years, several countries have deregulated and liberalized the entry of new public transport suppliers. Following the UK example (1980), there was a bus deregulation in Sweden (1997), Norway (2003) and more recently Germany (2013) as well as France (2015). ${ }^{1}$ Typically bus companies open a new direct line between two cities that were previously only connected indirectly via a rail network.

Engineers and economists have studied situations where adding a new route may decrease social welfare. ${ }^{2}$ This may only occur in the presence of unpriced congestion (or at least if congestion is not priced at the first best). As we will see, this natural conspiracy may occur also in the public transport context considered here. In this case the externality is the waiting time externality. We will study here the potentially negative consequences of changing the network (by adding a new service) or changing market organization (with the divestiture of two operators) in a public transport system. Often, when some new infrastructure is built, there are winners and losers (those may be compensated). We will use the term "destructive change" to refer to this extreme case where, after the change (of infrastructure or in organization) losers outweigh the gainers (social welfare decrease). In this case, loosers cannot be compensated.

We focus on the simplest set up of public transport links. Take any two public transport links that are connected $(\mathrm{AB}, \mathrm{BC})$ where part of the passengers also travel from $\mathrm{A}$ to $\mathrm{C}$ (see Fig. 1 for illustration). Then adding a direct service from $\mathrm{A}$ to $\mathrm{C}$ can increase the overall travel costs when the direct service is not managed by the same operator as the two connected lines. The two main ingredients are the positive effect derived from more passengers on a line as well as the absence of cooperation in the operation of the public transport lines. The positive externality associated with a more frequent bus or rail service when the number of passengers increases is attributed to Mohring (1972). Mohring's best known finding is that minimizing the sum of travel costs and bus operating costs, in a regime with frequent service

\footnotetext{
${ }^{1}$ For a review of the bus regulation in Europe see Van de Velde (2009).

${ }^{2}$ This is sometime referred to as the Braess paradox, in the context of private transportation
} 
and buses that are not full, implies that the frequency of service increases with the square root of the number of passengers. As waiting time is a component of the travel costs, an increase in the number of passengers will in general decrease waiting costs and travel costs: there is a positive externality associated with an increase of the density of passengers on a line. The second component is the lack of coordination between the initial lines (AB,BC) and the new line. When it would be the same operator, he would take into account the loss of surplus for the passengers that remain on the initial lines. But when it is a different operator, he will not be concerned about the loss of passengers on the other lines.

The issue we signal is more widespread than it appears at first sight. Pickrell (1990) found for the public transport networks of Buffalo, Miami and Sacramento that adding a link to the existing public transport network decreased overall ridership for public transport. Trips were probably diverted to the road network. In Europe, where public transport has a larger market share, the issue is common when a HSR line is built next to an existing rail line. HSR lines are often operated by a different operator (Thalys, Eurostar) than the local

rail lines. Whenever a new air transport link, or bus link, that competes with a network of rail lines is offered, the issue may also appear since the two suppliers are clearly different. The entry of new bus lines in the US and Germany is discussed in more detail in Augustin et al. (2014). Aarhaug \& Fearnley (2016) conclude that in Norway competition is essentially intermodal. de Palma et al. (2011) deserve several chapters to competition and regulation of transportation activities for different modes.

The paper is organized as follows: Section 2 presents the setting of the network, Section 3 shows under what condition the destructive change problem may occur and the extension to the case of synchronized services is discussed in Section 4. Section 5 presents illustrations and Section 6 concludes with caveats. Proofs and technical material are relegated to Appendices.

\section{The setting}

The paper considers a public transport network without congestion where a fixed number of passengers want to go from $\mathrm{A}$ to $\mathrm{B}$, from $\mathrm{B}$ to $\mathrm{C}$ and from $\mathrm{A}$ to $\mathrm{C}$ via $\mathrm{B}$. We show under 
what conditions the addition of a new direct line $\mathrm{AC}$, which avoids the use of the $\mathrm{AB}$ and BC links, would increase total user and operator costs. This can be relevant for any network where the additional line $\mathrm{AC}$ is operated by an operator other than that of the $\mathrm{AB}$ and $\mathrm{BC}$ lines.

Consider the transit system represented in Fig. 1. There are three stations $A, B$ and $C$ and three groups of users. Each group of users is identified by its origin-destination pair, denoted $(A B),(B C)$ and $(A C)$. Initially, there are only two transit lines $(A B)$ and $(B C)$. We distinguish between two management regimes. In the decentralized regime one operator manages lines $(A B)$ and $(B C)$ and a second operator manages line $(A C)$. In the centralized regime the same operator manages the three lines $(A B),(B C)$ and $(A C)$. The decentralized regime is common when group $(A C)$ is using a different mode. Then there are no technological economics of scope. Many regulators may favor the entry of a different operator because they fear abuse of monopoly power by the incumbent operator. We assume that the willingness to pay for the trip is the same for all passengers and is sufficiently high to make it worthwhile to organize a public transport service. The destructive change problem we discuss is consistent with two types of operator behavior: either he minimizes the sum of the user costs and the operator costs or he maximizes profits. In the first case, we are in the traditional Mohring case where the operator is instructed by a regulator to use this objective function. In the second case, the operator maximizes his profits for a given maximum willingness to pay and for a given ridership, by minimizing again the sum of user costs and operator costs and charging the maximum willingness to pay minus a small amount. $^{3}$

\footnotetext{
${ }^{3}$ When a second operator supplies services on link $\mathrm{AC}$ and competes with the operator of lines $\mathrm{AB}$ and $\mathrm{BC}$, it can be shown that if the destructive change problem appears, the user cost for passengers that use the direct $\mathrm{AC}$ link is always lower than the user cost of the $\mathrm{AB}+\mathrm{BC}$ link. With a lower user cost, the second operator will also, in the profit maximizing case, supply the direct link, and the initial operator cannot drive him out of the market with lower prices as the user cost of the initial operator is higher when the problem applies. This holds certainly for the case where users are uniform and when no price discrimination is possible.
} 


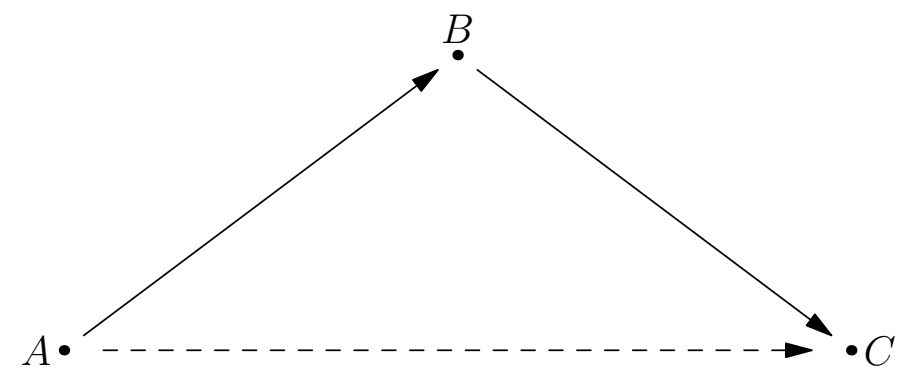

Figure 1: The existing transit system $\mathrm{AB}, \mathrm{BC}$ and the new entry $\mathrm{AC}$ - this setting is called $\mathscr{T}$

At the heart of the problem is the wait time. Assuming that users arrive at a uniform rate at the station, their average waiting time is equal to one divided by twice the frequency. The assumption of uniform arrival rates is widely accepted when the bus or train frequency is quite high. So, the wait time decreases as the service frequency increases and vice versa. ${ }^{4}$ With only two transit lines, users $(A C)$ need to commute through $B$. They have a longer travel time, and the new transit line project will a priori decrease the travel cost of these users, if line $(A C)$ is run by an operator who does not take externalities on $(A B)$ and $(B C)$ into account.

It is possible that in the initial case, the demand for the two lines $(A B)$ and $(B C)$ is high. The operator then sets relatively high frequencies. With the addition of the line $(A C)$, the demand on transit lines $(A B)$ and $(B C)$ declines, and the operator decreases the frequencies. The waiting time increases for users $(A B)$ and $(B C)$. Only users $(A C)$ may potentially benefit from the new transit line. We show that the net social impact is not necessarily positive. This result obtains since, initially, the public transport system benefits from economics of density which decrease when a new line $(A C)$ is operated by another

\footnotetext{
${ }^{4}$ When the service frequency is small, users are likely to consider timetables and incur a small or no wait time. Instead of wait time, one can consider a dynamic model with preferred arrival times and can still produce similar economics of density in the model. Intuitively, assume that users have uniformly distributed arrival times over a finite interval. When they use a train they will incur a schedule delay (either early or late arrival). We adopt below a general formulation that allows us to easily switch between wait time costs and schedule delay cost.
} 
operator, and users are more dispersed over the network provided all the links are used. In the next section, we prove this result using an analytical model. Notice that investment or construction costs are not taken into account (or assumed zero). However, the destructive change problem presents itself whether operating costs are included or not.

\section{The potential negative effects of entry}

We denote the transit system of Fig. 1 by $\mathscr{T}$. We denote by $n_{i j}$ the number of passengers travelling from node $i$ to node $j$. The number of travellers is kept fixed. We have, $n_{i j}>0$ for $i j=A B, B C$ and $A C$. Initially, there are only two transit lines $(A B)$ and $(B C)$, and the addition of a new direct line $(A C)$ is considered later. Let $f_{i j}$ denote the frequency of service on line $(i j)$, and let $t_{i j}$ denote the travel time from $i$ to $j$. For each passenger, the total travel time is the sum of waiting time and travel time. While nondeterministic services (cf. Monchambert \& de Palma 2014) can be assumed for a more realistic model, they do not affect our main conclusion but introduce further complications. So we assume that timetables are deterministic. The average waiting time for users of transit line $(i j)$ is $^{5}$ $\delta / f_{i j}$

\footnotetext{
${ }^{5}$ As we have mentioned in footnote 4 , we can either have a high frequency where users arrive at a uniform rate to the station, or a small frequency where the users use the time table, and do not incur wait time cost, but do incur schedule delay cost. In the former case, the average wait time is $1 / 2 f$, where $f$ is the service frequency. In the second case, denote the early arrival $\operatorname{cost} \beta$, the late arrival cost $\gamma$ and let the considered time period be normalized to one. The average schedule delay cost in this case is either $1 / 2 f$ in the case of high frequency or $\beta \gamma /(2(\beta+\gamma) f)$ in the case of low service frequencies and schedule costs. So, $\delta$ equals either $1 / 2$ (high frequency) or $\delta=(\beta \gamma) /(2(\beta+\gamma))$. Of course, $\delta$ may take other values to combine both effects. We normalize all unit costs so that the value of time equals 1.
} 
The total user cost, denoted $C_{1}$, is given by ${ }^{6}$

$$
C_{1}=\left(t_{A B}+\delta / f_{A B}\right)\left(n_{A B}+n_{A C}\right)+\left(t_{B C}+\delta / f_{B C}\right)\left(n_{B C}+n_{A C}\right),
$$

where users $(A C)$ necessarily select first $(A B)$ then $(B C)$. Notice that with two transit lines, users $(A C)$ board line $(A B)$ and then line $(B C)$.

The operating cost of a vehicle is constant and equal to $\theta$. The constant cost helps us to derive the results easily, but is not crucial for our conclusion. What matters is to have a total cost that increases with respect to the number of vehicles in service. The total cost in the system is the sum of the user's cost and the operator's cost, or

$$
T C_{1}=C_{1}+\theta \cdot\left(f_{A B}+f_{B C}\right)
$$

Notice that passengers $(A C)$ have a waiting time in station $A$ and a waiting time in station $B$. We assume that the two services $(A B)$ and $(B C)$ are not synchronized. ${ }^{7}$ As we show in Section 4, the main result also holds with synchronized service. We also assume that operation costs are sufficiently low so that it is never interesting to use vehicles at full capacity. This rules out a solution where public transport is congested, and we can concentrate on the internal solutions of the of Mohring problem where vehicles are never full.

The operator minimizes total travel cost $T C_{1}$. Taking the first-order conditions for (2) with respect to the frequencies and solving yields $f_{A B}=\sqrt{\delta\left(n_{A B}+n_{A C}\right) / \theta}$ and $f_{B C}=$ $\sqrt{\delta\left(n_{B C}+n_{A C}\right) / \theta}$. We check that second-order conditions are positive, so these solutions are global minima. These expressions are the well-known square root rule formula Mohring (1972). Plugging these frequencies in (1) and (2) yields, for the user cost,

$$
C_{1}^{*}=\left(n_{A B}+n_{A C}\right) t_{A B}+\left(n_{B C}+n_{A C}\right) t_{B C}+\sqrt{\theta \delta}\left(\sqrt{n_{A B}+n_{A C}}+\sqrt{n_{B C}+n_{A C}}\right)
$$

\footnotetext{
${ }^{6}$ We assume that all alternatives have the same fares for the user. Price competition between operators leads to complex analysis. We have an ongoing research project that addresses this particular issue. Baker \& White (2010) have noticed that, even at the empirical level, the impacts of fare changes on operators is difficult to measure.

${ }^{7}$ So, at station $B$, users $(A C)$ have the same average wait time as users $(B C)$.
} 
and for the total cost we have

$$
T C_{1}^{*}=C_{1}^{*}+\sqrt{\theta \delta}\left(\sqrt{n_{A B}+n_{A C}}+\sqrt{n_{B C}+n_{A C}}\right) .
$$

The stars denote optimized quantities with respect to frequencies. $\theta \delta$ is found in several expressions. Notice that $\theta$ is a technological cost parameter, while $\delta$ is a behavioral cost parameter.

Now consider the case where an additional line $(A C)$ is available. Each group of passengers will use its own transit line. Then total user cost is the sum of the cost for each group. It is given by

$$
C_{2}=\left(t_{A B}+\delta / f_{A B}\right) n_{A B}+\left(t_{B C}+\delta / f_{B C}\right) n_{B C}+\left(t_{A C}+\delta / f_{A C}\right) n_{A C}
$$

and total travel time is obtained, similarly as for Eq. (2), by adding operators costs, i.e.

$$
T C_{2}=C_{2}+\theta \cdot\left(f_{A B}+f_{B C}+f_{A C}\right)
$$

The square root rule holds again for each transit line and we have $f_{i j}=\sqrt{\delta n_{i j} / \theta}$ for $i j=$ $A B, B C, A C$. Plugging the values of these frequencies in the expressions above yields for the user cost

$$
C_{2}^{*}=n_{A B} t_{A B}+n_{B C} t_{B C}+n_{A C} t_{A C}+\sqrt{\theta \delta}\left(\sqrt{n_{A B}}+\sqrt{n_{B C}}+\sqrt{n_{A C}}\right),
$$

and for the total cost

$$
T C_{2}^{*}=C_{2}^{*}+\sqrt{\theta \delta}\left(\sqrt{n_{A B}}+\sqrt{n_{B C}}+\sqrt{n_{A C}}\right) .
$$

For the discussion below it is simpler to start from the following case.

Assumption 1 (symmetry). The travel cost of the new line is the sum of the travel costs of the two existing lines, i.e. $t_{A C}=t_{A B}+t_{B C}$.

To evaluate the impact of the new line we have the choice whether to include or not the cost of the operator. We show below that in both cases it is possible that the problem arises. We have the following result: 
Lemma 1. Consider the transit system $\mathscr{T}$ and decentralized management of the lines. Then, under Assumption 1, the variation of total cost after adding the new line has the same sign as the variation of user costs:

$$
\frac{1}{2}\left(T C_{2}^{*}-T C_{1}^{*}\right)=C_{2}^{*}-C_{1}^{*}=\sqrt{\theta \delta} \cdot \psi\left(n_{A B}, n_{B C}, n_{A C}\right),
$$

where

$$
\psi\left(n_{A B}, n_{B C}, n_{A C}\right) \equiv \sqrt{n_{A C}}-\left(\sqrt{n_{A C}+n_{A B}}-\sqrt{n_{A B}}\right)-\left(\sqrt{n_{A C}+n_{B C}}-\sqrt{n_{B C}}\right) .
$$

Furthermore, for any $n_{A B}, n_{B C}>0$, let $\hat{n}_{A C}>0$ be the unique solution to $\psi\left(n_{A B}, n_{B C}, n_{A C}\right)=$ 0 in $n_{A C}$. Then, $\psi\left(n_{A B}, n_{B C}, n_{A C}\right)>0$ if, and only if, $0<n_{A C}<\hat{n}_{A C}$.

The proofs are given in Appendix A. The first part in Lemma 1 states that whether the impact of the new transit line $(A C)$ is positive or negative does not depend on whether the operator's cost has been included (in the total cost) or not. Without the operator's cost, the magnitude of the impact of the new transit line is half as important. The second part of the lemma says that for any sizes of user groups $(A B)$ and $(B C)$, it is possible to find a sufficiently small, but positive, size of group $(A C)$ such that the new line induces an increase in the total cost. It is then important to check under what conditions this new line will indeed be used by group $(A C)$.

Lemma 2 (Users choice). Under Assumption 1, when users $(A C)$ have the choice between path $(A B),(B C)$ or path $(A C)$, they all select path $(A C)$ if, and only if, $n_{A C}>\hat{n}_{A C}$, where $\hat{\hat{n}}_{A C}$ is the unique solution of $\phi\left(n_{A B}, n_{B C}, n_{A C}\right)=0$, in $n_{A C}$, with

$$
\phi\left(n_{A B}, n_{B C}, n_{A C}\right)=n_{A C}^{-1 / 2}-\left(n_{A C}+n_{A B}\right)^{-1 / 2}-\left(n_{A C}+n_{B C}\right)^{-1 / 2} .
$$

This result shows that when the number of passengers from $A$ to $C$ is sufficiently large they choose the new direct transit line $(A C)$ and no longer transit through $B$. This is because, when $n_{A C}$ is large, the operator of the new transit line sets high frequency, leading to smaller wait time on line $(A C)$. The next step is to check that the results of Lemma 1 and Lemma 2 can be obtained simultaneously. 
Lemma 3. Let $\hat{n}_{A C}$ and $\hat{\hat{n}}_{A C}$ be as defined in Lemma 1 and Lemma 2, respectively, i.e. $\psi\left(n_{A B}, n_{B C}, \hat{n}_{A C}\right)=\phi\left(n_{A B}, n_{B C}, \hat{n}_{A C}\right)=0$. Then, for all $n_{A B}, n_{B C}>0$, we have $\hat{\hat{n}}_{A C}<\hat{n}_{A C}$.

It is now straightforward to state the main result.

Proposition 1 (Symmetry: $\left.t_{A C}=t_{A B}+t_{B C}\right)$. Consider the transit system $\mathscr{T}$ and Assumption 1. If a new operator runs the new line $(A C)$ then it is used by group $(A C)$ and it increases total user cost if, and only if, $\hat{\hat{n}}_{A C}<n_{A C}<\hat{n}_{A C}$, where $\hat{n}_{A C}$ and $\hat{\hat{n}}_{A C}$ are defined in Lemma 1 and Lemma 2, respectively.

From Proposition 1, when the transit system is operated by distinct agents it is possible that a new transit line is added to the network and has customers while it leads to an increase in the average user cost. When all the transit lines are operated by a single agent, only a naive decision process will lead to such network development, since the global optimum will always select the solution with the lowest cost.

Consider the symmetric case (Assumption 1), and let variables $x$ and $y$ be defined as proportion of passengers that want to go from $\mathrm{A}$ to $\mathrm{B}$, and $\mathrm{B}$ to $\mathrm{C}$ relative to passengers that want to go from A to C: i.e. $x=n_{A B} / n_{A C}$ and $y=n_{B C} / n_{A C}$. It is clear then, that the sign of $\psi\left(n_{A B}, n_{B C}, n_{A C}\right)$ depends only on $x$ and $y$. In the $(x, y)$-plane, the implicit curve defined by equation $\psi\left(n_{A B}, n_{B C}, n_{A C}\right)=0$ is denoted by $\mathscr{C}_{1}$. Fig. 2 is given for illustration. The destructive change problem, described in Proposition 1, appears only in the area above $\mathscr{C}_{1}$ (parts II and III). On the same figure, curve $\mathscr{C}_{2}$ corresponds to Eq. (11) in the $(x, y)$-plane. From Lemma 2, the new transit line $(A C)$ is the choice of users $(A C)$ only for values of $x$ and $y$ below $\mathscr{C}_{2}$ (areas I and II). For $x$ and $y$ in area III, above curve $\mathscr{C}_{2}$, users $(A C)$ will prefer path $(A B),(B C)$ because it has a smaller user cost.

With decentralized management of the transit system, an operator will operate line $(A C)$, and users $(A C)$ will use it, but the overall user cost may increase due to the increase in wait time for users $(A B)$ and users $(B C)$. Next, we show that the main result may hold also in the asymmetric case (when Assumption 1 is not satisfied). 


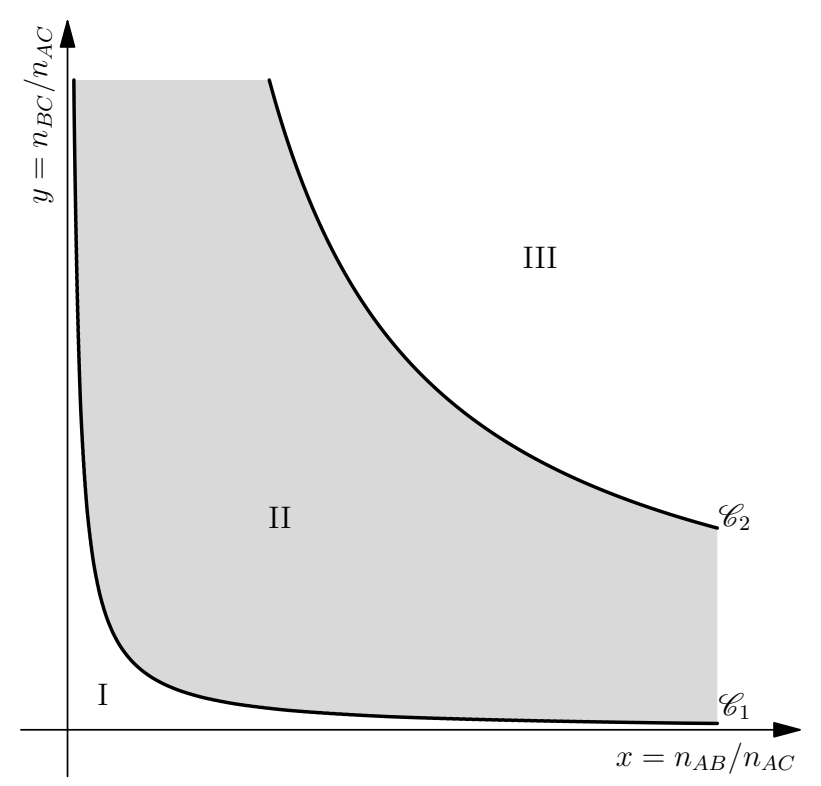

Figure 2: in zone II the new line $\mathrm{AC}$ increases total average cost of the system

Proposition 2 (Non symmetry: $t_{A C} \lessgtr t_{A B}+t_{B C}$ ). Let $\Delta t=t_{A B}+t_{B C}-t_{A C}$ and define $\hat{\Delta t}=\sqrt{\theta \delta} \psi\left(n_{A B}, n_{B C}, n_{A C}\right) / n_{A B}$ and $\hat{\Delta} t=\sqrt{\theta \delta} \phi\left(n_{A B}, n_{B C}, n_{A C}\right)$. When the new line $(A C)$ is run by a new operator, then (i) it decreases the user cost if $\Delta t>\hat{\Delta t}$, and increases user cost if $\Delta t<\hat{\Delta t}$, (ii) it is used by group $(A C)$ if, and only if, $\Delta t>\hat{\Delta t}$. Moreover, for all $n_{A B}, n_{B C}, n_{A C}>0$, we have $\hat{\Delta} t<\hat{\Delta t}$.

On a general ground, the result in Proposition 2 is a direct consequence of the continuity of the cost functions and the Weierestrass theorem. It is interesting to illustrate the interplay between total users cost and the equilibrium condition for users $(A C)$. In Section 5 below, we provide an illustration with non symmetric parameter values, i.e. with $t_{A C} \lessgtr t_{A B}+t_{B C}$. Notice that even for $t_{A C}>t_{A B}+t_{B C}$ line $(A C)$ may still be the choice of users $(A C)$ because it has a smaller waiting time than path $(A B),(B C)$. This can occur when users $(A C)$ have a wait time cost in station $B$, but it is ruled out when the same train travels from $A$ to $B$ to $C$. The formal analysis of this case is given in Section 4, but the intuition for the result is quite easy to see. Indeed, when users $(A C)$ do not have a wait time in station $B$, and if $t_{A C}>t_{A B}+t_{B C}$ they will benefit from line $(A C)$ only when their wait time in station $A$ decreases, and thus, only when the frequency of the new transit line is higher than the 
frequency on path $(A B),(B C)$. This is not possible since the frequency is increasing with the number of users (see the square root rules derived above) and users $(A C)$ no longer benefit from the positive impact of users $(A B)$ and users $(B C)$.

As a last point in this section we ask whether a voting process can lead to the destructive change problem we discuss in this paper. The following result provides a negative answer.

Proposition 3. (Majority voting) Let $\hat{\Delta t}$ and $\hat{\Delta t}$ be defined as in Proposition 2. Operating the new line $(A C)$ will be adopted by majority voting if, and only if, $n_{A C}>n_{A B}+n_{B C}$ and $\hat{\Delta} t<\hat{\Delta t}$. Moreover, when $n_{A C}>n_{A B}+n_{B C}$ we have $\hat{\Delta t}<0$.

Proposition 3 implies that when the median user benefits from the new transit line, then it is welfare improving and it leads to a decrease in the average user cost. In the symmetric case (Assumption 1), when $n_{A C}>n_{A B}+n_{B C}$ we necessarily have $\psi\left(n_{A B}, n_{B C}, n_{A C}\right) \leq 0$. In the next section we show that when services in lines $(A B)$ and $(B C)$ are synchronized, then the problem never appears under majority voting, independently of the values of $t_{A B}, t_{B C}$ and $t_{A C}$.

\section{Synchronized services}

When trains $(A B)$ and $(A C)$ are synchronized, users $(A C)$ have a smaller or no wait time at station $B$. In the simple case, they do not change platform when the same train departs from $A$, stops in $B$ and then continues to $C$. It follows that $f_{A B}=f_{B C}=f$ and that users $(A C)$ do not have a waiting time in station $B$, since they do not change train (we ignore dwell time at station $B$ ). The model is solved following the same procedure as in Section 3 .

Without transit line $(A C)$, replace all the frequencies with $f$ in Eqs. (1) and (2), and

minimize the total cost to find optimum frequency $f=\sqrt{\delta\left(n_{A B}+n_{B C}+n_{A C}\right) / \theta}$. Then, substitute into the total cost to find

$$
C_{1}^{*}=\left(n_{A B}+n_{B C}\right) t_{A B}+\left(n_{B C}+n_{A C}\right) t_{B C}+\sqrt{\theta \delta} \sqrt{n_{A B}+n_{B C}+n_{A C}} .
$$

With transit line $(A C)$, the frequencies are $f=\sqrt{\delta\left(n_{A B}+n_{B C}\right) / \theta}$ and $f_{A C}=\sqrt{\delta n_{A C} / \theta}$, 
and the total cost is

$$
C_{2}^{*}=n_{A B} t_{A B}+n_{B C} t_{B C}+n_{A C} t_{A C}+\sqrt{\theta \delta n_{A C}} \sqrt{\left(n_{A B}+n_{B C}\right) \theta \delta} .
$$

It follows that the difference in the total average user cost satisfies

$$
\frac{C_{2}^{*}-C_{1}^{*}}{\theta \delta n_{A C}}=1+\sqrt{\frac{n_{A B}+n_{B C}}{n_{A C}}}-\sqrt{\frac{n_{A B}+n_{B C}+n_{A C}}{n_{A C}}}-\Delta t \sqrt{\frac{n_{A C}}{\delta \theta}} .
$$

Comparing the users cost for group $(A C)$, we see that they will benefit from the new transit line and use it if, and only if,

$$
n_{A C}^{-1 / 2}-\left(n_{A B}+n_{B C}+n_{A C}\right)^{-1 / 2}-\Delta t / \sqrt{\delta \theta} \leq 0 .
$$

A small change of parameter values in the numerical example of Section 5 shows that a positive quantity in Eq. (12) and inequality (13) can hold simultaneously, yielding the problem of interest for the case of synchronized services. It is clear from Eq. (13) that, for $n_{A B}+n_{B C}>0$, the new transit line will be used only if $\Delta t>0$, i.e. $t_{A C}<t_{A B}+t_{B C}$. We then state the equivalent result of Proposition 3 for the case where transit services are synchronized.

Proposition 4. When services between lines $(A B)$ and $(B C)$ are synchronized, the destructive change problem never appears under majority voting when all users participate.

When train services are not synchronized we have shown that voting can lead to the issue

of interest, but only for some cases where line $(A C)$ is longer than the sum of the length of lines $(A B)$ and $(B C)$ (cf. Proposition 3). Proposition 4 shows that this can never hold independently of the length of the three lines. The intuition of this result is quite simple. Indeed, when services are synchronized, users $(A C)$ will use line $(A C)$ only when it is shorter than the sum of the length of the two other lines, while in the absence of synchronization, they may switch to a longer line $(A C)$ to avoid wait time at $B$.

\section{$5 \quad$ Illustration and policy implications}

It is helpful to use first a small numerical example before moving on to some real world examples. Table 1 provides the parameter values. The first two lines provide the input data 
(number of users and travel cost on each link). Under case I, there are only the two transit lines $(A B)$ and $(B C)$. Under case II, transit line $(A C)$ is added. For each case, we compute the frequencies, the waiting time and the average user cost for each group. In the input data, the transit line $(A C)$ is slightly shorter than the sum of the lengths of the two other transit lines. The average total cost is given in the last two columns. In column $C$ we use Eqs. (1) and (5), so the operator's cost is not included. In column TC, we use Eqs. (2) and (6), so the operator's cost is included. In the last block, the impact of operating the new transit line $(A C)$ is evaluated. In this case, we find that the service frequencies decrease for transit lines $(A B)$ and $(B C)$, so the average waiting times increase for the corresponding groups but decrease for the users of the new transit line. This is because they only wait in station $A$. The last line in the impacts block shows that benefits to users $(A C)$ from the new transit line is not large enough to compensate for the loss of users in the other two groups. In this example, both $C$ and $T C$ increase. Notice that in Table 1, the impact on the total cost when operating cost is included is more than twice the impact when only total user costs are taken into account. Indeed, Eq. (9) holds under Assumption 1, while in this example we have $t_{A C}<t_{A B}+t_{B C}$.

The numerical values in Table 1 yield a small increase in the user cost. One may ask whether this is always the case, or if it is possible to have other cases with large increase in users costs. To answer this question it is helpful to evaluate the upper bound on the change in the user cost caused by the new transit line. From Eq. (10), we have that $\psi\left(n_{A B}, n_{B C}, n_{A C}\right) \leq$ $\sqrt{n_{A C}}$. Then, using Eqs. (3) and (7) we get, for $t_{A C} \leq t_{A B}+t_{B C}$, the following inequality

$$
C_{2}^{*}-C_{1}^{*} \leq n_{A C}\left(\sqrt{\theta \delta / n_{A C}}-\Delta t\right)
$$

where $N=n_{A B}+n_{B C}+n_{A C}$, the total number of transit users, and $\Delta t=t_{A B}+t_{B C}-t_{A C}$. For the numerical values considered in this example, the upper bound on the impact is smaller than one $(\approx 0.80)$, but is not even reached since it requires that $n_{A B}$ and $n_{B C}$ are very large with respect to $n_{A C}$. This will at the same time, however, lead to increases in $N$ in Eq. (14). The only case where one can obtain large impacts is when $n_{A C}$ is very small and dominated by small values of $n_{A B}$ and $n_{B C}$. Indeed, in this case the right hand member 


\begin{tabular}{|c|c|c|c|c|c|}
\hline & \multicolumn{3}{|c|}{ Transit lines and users } & \multicolumn{2}{|c|}{$\operatorname{Costs}^{a}$} \\
\hline & $A B$ & $B C$ & $A C$ & $C$ & $T C$ \\
\hline \multicolumn{6}{|l|}{ Input data } \\
\hline Number of users & 25 & 25 & 10 & - & - \\
\hline In-vehicle travel time (mn) & 5 & 5 & 9.80 & - & - \\
\hline \multicolumn{6}{|c|}{ Case I: only transit lines $(A B)$ and $(B C)$} \\
\hline Frequencies (veh/mn) & 0.59 & 0.59 & - & - & - \\
\hline Average wait time (mn) & 0.84 & 0.84 & 1.68 & - & - \\
\hline Total average travel time (mn) & 5.85 & 5.85 & 11.69 & 6.82 & 7.80 \\
\hline \multicolumn{6}{|c|}{ Case II: three transit lines $(A B),(B C)$ and $(A C)$} \\
\hline Frequencies (veh/mn) & 0.50 & 0.50 & 0.32 & - & - \\
\hline Average wait time (mn) & 1.00 & 1.00 & 1.58 & & \\
\hline Total average travel time (mn) & 6.00 & 6.00 & 11.38 & 6.90 & 7.99 \\
\hline \multicolumn{6}{|l|}{ Impacts } \\
\hline on frequencies (veh/mn) & -0.09 & -0.09 & - & - & - \\
\hline on wait time $(\mathrm{mn})$ & 0.16 & 0.16 & -0.10 & - & - \\
\hline on total average travel time (mn) & 0.15 & 0.15 & -0.31 & 0.08 & 0.19 \\
\hline
\end{tabular}

${ }^{a}$ costs are computed on the assumption that one minute is equal to one monetary unit.

Table 1: An illustration of the destructive change problem 
in Eq. (14) will be proportional to $1 / \sqrt{n_{A C}}$ and can be made arbitrarily large by scaling down the number of users. It is clear that this remains a theoretical possibility. In Figure 3, we have set $n_{A C}=10$ and draw the contour levels for the impact on the average user costs resulting from new transit line for distinct values of $n_{A B}$ and $n_{B C} \cdot{ }^{8}$ Curves $\mathscr{C}_{1}^{\prime}$ and $\mathscr{C}_{2}^{\prime}$ do the same plane partition as curves $\mathscr{C}_{1}$ and $\mathscr{C}_{2}$, respectively, given in Fig. 1 . So, the issue arises in the area between $\mathscr{C}_{1}^{\prime}$ and $\mathscr{C}_{2}^{\prime}$. Each contour line corresponds to the indicated change in the average user cost, and the positive values indicate that the introduction of the transit line $(A C)$ increases the average user cost. The contour lines show that the maximum increase in the average user cost is obtained for finite values of $n_{A B}$ and $n_{B C}$ inside the curve of contour level 0.076 .

In practice, PT networks are more complex than three nodes and an extra more direct link is often provided using another mode. This can be a direct bus line, a HSR line or a flight added to national train service. The new line will then be quicker and sometimes more expensive, or slower and less expensive. It remains true that the addition of the new line decreases the generalized cost but with a heterogeneous population, not all customers may opt for the new alternative. We limit this paper to two illustrations. In the North of Belgium, Brussels used to be connected to the Rotterdam-Amsterdam area via a rather slow international train with a frequency of one train per hour. This train made more or less 10 stops in between. This international (slow) train has been replaced by a fast and more expensive train service (Thalys, international joint venture) that made only 3 stops between Brussels Midi and Amsterdam. This has resulted in a strongly deteriorated service for the smaller towns in between that see their frequency of service strongly reduced. This problem is probably less likely to occur when the same rail operator organizes the local trains as well as the faster more direct trains. A second example concerns the competition of the German railways by direct bus lines (Augustin et al. (2014)). The intercity rail service is organized by the Deutsche Bahn (DB) and up to 2012, competing intercity services were not allowed except if they offered a significantly better or cheaper service. No intercity buses (except

\footnotetext{
${ }^{8}$ Fig. 3 is similar to Fig. 2 except for the change of variables. Indeed, in Fig. 3 the $\left(n_{A B}, n_{B C}\right)-$ plane is used instead, and we consider the difference in cost averaged over the total number of users.
} 


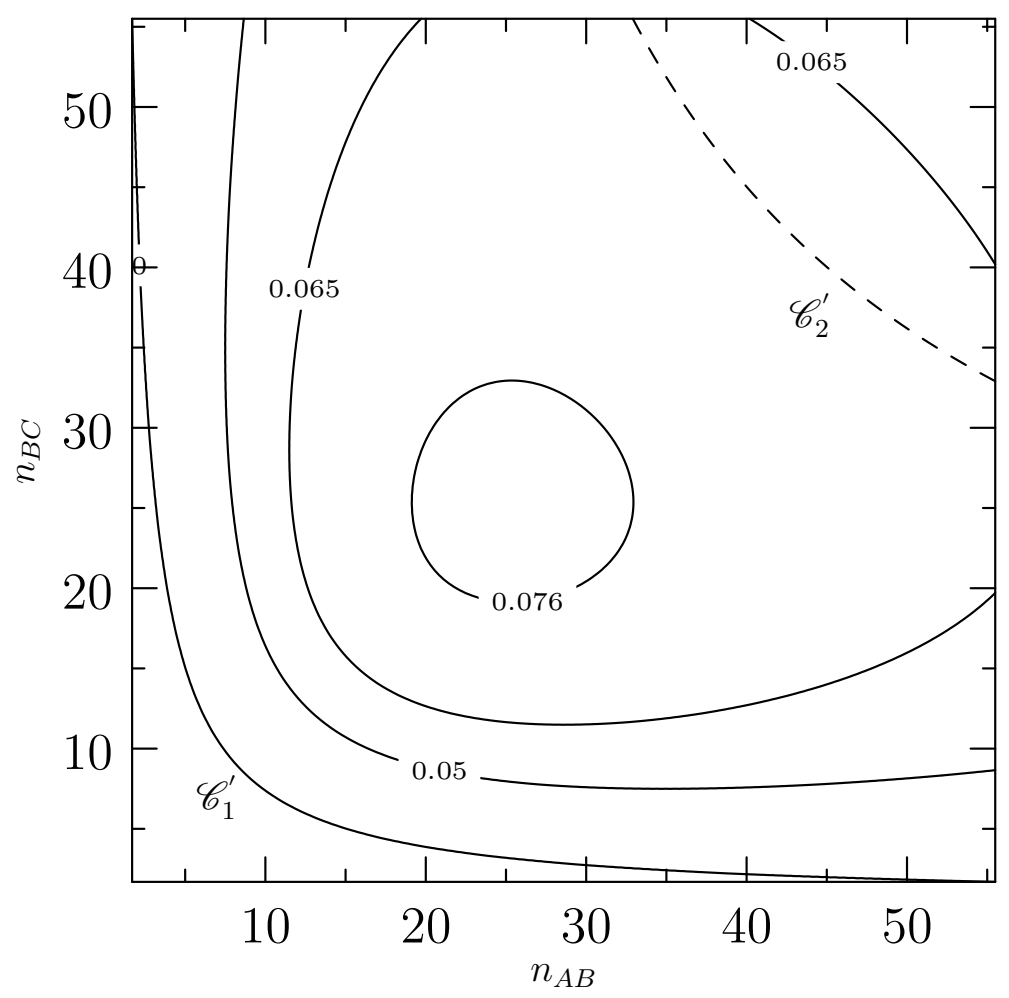

Figure 3: Contour levels showing the impact of the new transit line on the average user cost (for $n_{A C}=10$ ): above curves $\mathscr{C}_{1}^{\prime}$ the user cost increases and below curve $\mathscr{C}_{2}^{\prime}$ users $A C$ choose the new line $A C$ - the destructive change problem occurs in the zone between $\mathscr{C}_{1}^{\prime}$ and $\mathscr{C}_{2}^{\prime}$ (cf. Lemma 2) 
those controlled by DB) were operated. But in 2013, intercity buses were officially allowed and there are now several intercity bus companies and more than 100 new bus lines were created. According to DB, one third of the passengers of the new bus lines have switched away from intercity rail lines (Barrow (2014)). Bus transport is cheaper and can take more direct routes than rail, which is constrained to use the existing track infrastructure.

\section{Policy implications and conclusions}

What are the policy implications? In the destructive change problem we describe there is a conflict due to economics of density. Subsidizing the external economics of density for both alternatives $(A B, B C$ and $A C)$ will not lead to the right solution as the best solution is a corner solution. The only way to avoid the problem is to not allow a competing operator to open the new line and reserve the right for new lines to the incumbent operator. The issue may be stated in other forms to fit different situations. Consider, for example, the situation where transit lines $(A B)$ and $(A C)$ are operated by another (single) agent (or a country), say $A$, and transport line $(B C)$ is operated by an agent (a country), say $B$. The operators (countries) $A$ and $B$ do not cooperate and each one is concerned by the travel cost of its own users. If the number of users $(A B)$ is large enough, the introduction of the new transit line will not reduce the average user cost for groups $(A B)$ and $(B C)$. In this case operator $A$ of the network will not undertake the investment and users $(A B)$ will continue to benefit from the positive externality induced by users $(A C)$ on their line. However, if the number of users $(A B)$ is small in comparison to $(A C)$, then operator $A$ will choose to invest in the new line, and the positive externality for users $(A B)$ will be removed. The global impact, in this case, will depend on the size of group $(B C)$. If it is large enough we will have a global negative impact on the network.

In this paper we have described a situation where the expansion of a PT network can contribute to an increase in total user cost. This result suggests that a careful inspection of the network is needed before any investment decision is made. Indeed, mass transit benefits from the large number of users on each line. Our result has some similarity with the empirical 
analysis in Winston \& Maheshri (2007) who find that many urban rail systems in the US are not economically justified. Winston and Maheshri attribute the decrease in demand to urban sprawl and the induced lower density, and did not refer to the structure of the rail network itself as we do in this model. We also notice that our analysis disregards long run impacts and the possible relocation of households and firms induced by the development of transit network (cf. Baum-Snow et al. 2012). As users in A and C can be better off than users in $\mathrm{B}$, the direct link AC may very well imply a loss of population and business for location B. A full analysis of these second order effects requires a general equilibrium approach as suggested by Redding \& Turner (2015). Ahlfeldt et al. (2015) show how those towns that happen to be selected as HSR stop along the Cologne - Frankfort line, benefit from these second order relocation effects.

Finally our result was obtained using several assumptions. The most important one is that the operator will adjust the service frequency as a function of the number of users of each line. This was first stated in Mohring (1972) and is widely accepted by researchers and practitioners. Also, we have implicitly considered a single mode and inelastic demand. When other modes are involved their use may involve different types of externalities (road congestion, crowding) that may need additional consideration.

\section{Acknowledgement}

We are grateful to Richard Arnott, Winston Clifford and Émile Quinet for helpful comments. The usual disclaimer applies. 


\section{References}

Aarhaug, J. \& Fearnley, N. (2016), 'Deregulation of the norwegian long distance express coach market', Transport Policy 46, 1-6.

Ahlfeldt, G. M., Feddersen, A. et al. (2015), From periphery to core: Measuring agglomeration effects using high-speed rail, Discussion paper, Spatial Economics Research Centre, LSE.

Augustin, K., Gerike, R., Sanchez, M. J. M. \& Ayala, C. (2014), 'Analysis of intercity bus markets on long distances in an established and a young market: The example of the us and germany', Research in Transportation Economics 48, 245-254.

Baker, S. \& White, P. (2010), 'Impacts of free concessionary travel: Case study of an english rural region', Transport Policy 17(1), 20-26.

Barrow, K. (2014), 'Long-distance buses - the emerging challenge to germany's train operators', International Railway Journal (web journal) .

Baum-Snow, N., Brandt, L., Henderson, J. V., Turner, M. A. \& Zhang, Q. (2012), Roads, railroads and decentralization of chinese cities, Discussion paper, Brown University.

de Palma, A., Lindsey, R., Quinet, E. \& Vickerman, R. (2011), A handbook of Transport Economics, Edward Elgar Publishing.

Mohring, H. (1972), 'Optimization and scale economies in urban bus transportation', American Economic Review 62(4), 591-604.

Monchambert, G. \& de Palma, A. (2014), 'Public transport reliability and commuter strategy', Journal of Urban Economics 81, 14-29.

Pickrell, D. H. (1990), Urban rail transit projects: forecast versus actual ridership and costs: final report, Urban Mass Transportation Administration. 
Redding, S. J. \& Turner, M. A. (2015), Chapter 20 - Transportation costs and the spatial organization of economic activity, in J. V. H. Gilles Duranton \& W. C. Strange, eds, 'Handbook of Regional and Urban Economics', Vol. 5 of Handbook of Regional and Urban Economics, Elsevier, pp. $1339-1398$.

Van de Velde, D. (2009), Long-distance bus services in europe: concessions or free market?, Discussion paper, ITF-OECD 21.

Winston, C. \& Maheshri, V. (2007), 'On the social desirability of urban rail transit systems', Journal of Urban Economics 62(2), 362-382. 


\section{A Proofs}

\section{A.1 Proof of Lemma 1}

The expressions in Eqs. (9) and (10) are obtained by rearranging terms in Eqs. (3), (4), (7) and (8). Let $x=n_{A B} / n_{A C}$ and $y=n_{B C} / n_{A C}$. Taking $\sqrt{n_{A C}}$ as a factor in $\psi$, we can introduce function $g(x, y)=1+\sqrt{x}-\sqrt{1+x}+\sqrt{y}-\sqrt{1+y}$ so that $\psi\left(n_{A B}, n_{B C}, n_{A C}\right)=$ $\sqrt{n_{A C}} g(x, y)$. We have to show that $g(x, y)$ can take positive values for $x, y \geq 0$. Notice that $\sqrt{x}-\sqrt{1+x}$ is a strictly increasing function of $x$ from -1 (for $x=0$ ) to 0 (for $x \rightarrow \infty$ ). Taking into account the symmetry between $x$ and $y$ in $g(x, y)$, we immediately conclude that for sufficiently large $x$ and $y, g(x, y)$ is positive. From the definition of the variables, large $x$ and $y$ correspond to relatively small values of $n_{A C}$ with respect to $n_{A B}$ and $n_{B C}$. The continuity of $\psi$ implies the existence of threshold value $\hat{n}_{A C}$.

\section{A.2 Proof of Lemma 2}

We focus on the cost for users $(A C)$ under the two regimes. Without line $(A C)$ the average user cost for this group is $t_{A B}+t_{B C}+\sqrt{\theta \delta\left(n_{A B}+n_{A C}\right)}+\sqrt{\theta \delta\left(n_{B C}+n_{A C}\right)}$, and with line $(A C)$ their user cost becomes $t_{A C}+\sqrt{\theta \delta n_{A C}}$. Function $\phi$ is obtained by taking the difference and considering Assumption 1. The new line will be used if, and only if, it reduces the cost for users $(A C)$, i.e. if $\phi\left(n_{A B}, n_{B C}, n_{A C}\right)<0$. Using the same notation in the proof of Lemma 1 , we can introduce $h(x, y)=1-1 / \sqrt{1+x}-1 / \sqrt{1+y}$, in order to express $\phi\left(n_{A B}, n_{B C}, n_{A C}\right)=h(x, y) / \sqrt{n_{A C}}$. It is then clear that $h(x, y)<0$ when $x$ and $y$ are

sufficiently small. So, $\phi\left(n_{A B}, n_{B C}, n_{A C}\right) \leq 0$ when $n_{A C}$ is relatively large with respect to $n_{A B}$ and $n_{B C}$. The existence of threshold value $\hat{\hat{n}}_{A C}$ follows from the monotonicity and continuity of $\phi$ in $n_{A C}$.

\section{A.3 Proof of Lemma 3}

Consider $x, y, g(x, y)$ and $h(x, y)$ defined in the proofs of Lemma 1 and Lemma 2. We have to prove that the intersection of the set $\{(x, y)>0$ such that $g(x, y)>0\}$ and the set 
$\{(x, y)>0$ such that $h(x, y)<0\}$ is nonempty. We can show that $g(x, y)>0$ if, and only if, $y<y_{\psi}(x)$ where

$$
y_{\psi}(x)=\frac{-4(\sqrt{x+1}-1) x^{3 / 2}+4 x^{2}-4 x \sqrt{x+1}+x+(\sqrt{x+1}+1) \sqrt{x}+\sqrt{x+1}+1}{8 x} .
$$

Similarity, $h(x, y)<0$ if, and only if, $y>y_{\phi}(x)$ where

$$
y_{\phi}(x)=\left(2+3 x+(1+x)^{3 / 2}\right) / x^{2} .
$$

Some algebra shows that for all $x>0$, we have $y_{\phi}(x)<y_{\psi}(x)$, which yields the desired nonempty intersection.

\section{A.4 Proof of Proposition 2}

Notice that $t_{i j}$ enters the expression of the total (or user) cost additively. By continuity of the cost function, if $t_{A C}$ is slightly higher or smaller than the sum of $t_{A B}$ and $t_{B C}$ the new transit line will also lead to an increase in the total transport cost. We have to prove that $\hat{\Delta t}<\hat{\Delta}$. By using $x=n_{A B} / n_{A C}$ and $y=n_{B C} / n_{A C}$ this is equivalent to showing that for all $x, y>0$ we have $h(x, y)<g(x, y)$. Using the symmetry in these functions it suffices to show that $\sqrt{1+x}-\sqrt{x}<1 / \sqrt{1+x}$. Let $X=\sqrt{1+x}$ and notice that for $x>0$ we have $X>1$. This inequality becomes $X-\sqrt{X^{2}-1}<1 / X$. Taking the square root term alone on one side and computing the square on both sides the inequality simplifies to $1 / X^{2}<1$, which is true for all $X>1$.

\section{A.5 Proof of Proposition 3}

Users $(A B)$ and users $(B C)$ always vote against the project since it leads to a decrease in the frequency and hence in their wait time. Users $(A C)$ vote for the project only when it decreases their generalized travel cost (sum of wait time and travel time). This states the

first part of the proposition. Using the same notation as in the proof of Proposition 1, from Eqs. (3) and (7) we have

$$
\left(C_{2}^{*}-C_{1}^{*}\right) \sqrt{\frac{1}{\delta \theta n_{A C}}}=g(x, y)-\sqrt{\frac{n_{A C}}{\delta \theta}} \Delta t .
$$


Inequality $n_{A C}>n_{A B}+n_{B C}$ is equivalent to $x+y<1$. By solving the nonlinear program: $\max _{x, y \geq 0} g(x, y)$ under the constraint $x+y \leq 1$, we check that for $x, y \geq 0$ and $x+y \leq 1$, we have $g(x, y) \leq 1+\sqrt{2}-\sqrt{6}<0$. Thus, for $\Delta t \geq 0$ and $n_{A B}+n_{B C} \leq n_{A C}$ we have $\Delta C \leq 0$, i.e. the new transit line $(A C)$ necessarily decreases the total user cost. 